\title{
SQUARE FUNCTIONS OF FRACTIONAL HOMOGENEITY AND WOLFF POTENTIALS
}

\author{
VASILIS CHOUSIONIS, LAURA PRAT AND XAVIER TOLSA
}

\begin{abstract}
In this paper it is shown that for any measure $\mu$ in $\mathbb{R}^{d}$ and for a non-integer $0<s<d$, the Wolff energy $\iint_{0}^{\infty}\left(\frac{\mu(B(x, r))}{r^{s}}\right)^{2} \frac{d r}{r} d \mu(x)$ is comparable to$$
\iint_{0}^{\infty}\left(\frac{\mu(B(x, r))}{r^{s}}-\frac{\mu(B(x, 2 r))}{(2 r)^{s}}\right)^{2} \frac{d r}{r} d \mu(x),
$$

unlike in the case when $s$ is an integer. We also study the relation with the $L^{2}$-norm of $s$-Riesz transforms, $0<s<1$, and we provide a counterexample in the integer case.

\section{INTRODUCTION}

Let $\mu$ be a Radon measure in $\mathbb{R}^{d}$. Given $x \in \mathbb{R}^{d}, r>0$ and $s>0$, we set

$$
\Delta_{\mu}^{s}(x, r):=\frac{\mu(B(x, r))}{r^{s}}-\frac{\mu(B(x, 2 r))}{(2 r)^{s}} .
$$

The main result of this paper shows the comparability between the squared $L^{2}(\mu)$-norm of a square function involving the difference of densities (1.1) and the Wolff energy of the measure $\mu$, for measures $\mu$ in $\mathbb{R}^{d}$ and non-integer $s, 0<s<d$. Before stating precisely the theorem, we need to introduce some notation.

Let $\theta_{\mu}^{s}(B(x, r))$ be the average $s$-dimensional density of $\mu$ on $B(x, r)$, that is

$$
\theta_{\mu}^{s}(B(x, r))=\frac{\mu(B(x, r))}{r^{s}}
$$

so that

$$
\Delta_{\mu}^{s}(x, r)=\theta_{\mu}^{s}(B(x, r))-\theta_{\mu}^{s}(B(x, 2 r)) .
$$

Let $\alpha>0$ and $p \in(0, \infty)$ such that $\alpha p \in(0, d)$. The Riesz capacity $\dot{C}_{\alpha, p}$ of $E \subset \mathbb{R}^{d}$ is defined as

$$
\dot{C}_{\alpha, p}(E)=\sup _{\mu \in M(E)}\left(\frac{\mu(E)}{\left\|I_{\alpha} * \mu\right\|_{p^{\prime}}}\right)^{p}, \quad I_{\alpha}(x)=\frac{A_{d, \alpha}}{|x|^{d-\alpha}},
$$

where $M(E)$ is the set of positive Radon measures supported on $E$ and as usual $p^{\prime}=$ $p /(p-1)$. In nonlinear potential theory Riesz capacities occur naturally in the study of Sobolev spaces, for example they measure exceptional sets for functions in these function spaces, see e.g $[\mathrm{AH}]$.

V.C. was funded by the Academy of Finland Grant SA 267047. Also, partially supported by the ERC Advanced Grant 320501, while visiting Universitat Autònoma de Barcelona. L.P. and X.T. were partially supported by the grants MTM-2013-44304-P (MICINN, Spain) and 2014-SGR-75 (Catalonia). X.T. was also supported by the ERC grant 320501 of the European Research Council (FP7/2007-2013) and by Marie Curie ITN MAnET (FP7-607647). 
For $\alpha$ and $p$ as before the Wolff potential of a positive Radon measure $\mu$ is defined as

$$
\dot{W}_{\alpha, p}^{\mu}(x)=\int_{0}^{\infty}\left(\frac{\mu(B(x, r))}{r^{d-\alpha p}}\right)^{2} \frac{d r}{r}, \quad x \in \mathbb{R}^{d},
$$

and its Wolff energy is

$$
\int \dot{W}_{\alpha, p}^{\mu}(x) d \mu(x)
$$

Riesz capacities can be characterized via Wolff potentials, as a well known theorem of Wolff, see e.g. [AH, Theorem 4.5.4], asserts that

$$
C^{-1}\left\|I_{\alpha} * \mu\right\|_{p^{\prime}}^{p^{\prime}} \leq \int \dot{W}_{\alpha, p}^{\mu}(x) d \mu(x) \leq C\left\|I_{\alpha} * \mu\right\|_{p^{\prime}}^{p^{\prime}}
$$

where $C$ is a constant depending only on $d, \alpha$ and $p$.

In this paper we consider Wolff potentials with indices $\frac{2}{3}(d-s), \frac{3}{2}$, where $0<s<d$. Notice that Wolff potentials with these choice of indices are related to the $s$-dimensional density of $\mu$, in particular

$$
\dot{W}_{\frac{2}{3}(d-s), \frac{3}{2}}^{\mu}(x)=\int_{0}^{\infty}\left(\frac{\mu(B(x, r))}{r^{s}}\right)^{2} \frac{d r}{r}=\int_{0}^{\infty} \theta_{\mu}^{s}(B(x, r))^{2} \frac{d r}{r}, \quad x \in \mathbb{R}^{d} .
$$

We further remark that these potentials are related to the Calderón-Zygmund capacities associated with the vector valued Riesz kernels $K^{s}(x)=x /|x|^{1+s}, x \in \mathbb{R}^{d}$, see e.g. the excellent survey [EV2] or [MPV].

Our main result reads as follows:

Theorem 1.1. Let $\mu$ be a Radon measure on $\mathbb{R}^{d}$ and $0<s<d$ be non-integer. Then

$$
\iint_{0}^{\infty} \Delta_{\mu}^{s}(x, r)^{2} \frac{d r}{r} d \mu(x) \approx \int \dot{W}_{\frac{2}{3}(d-s), \frac{3}{2}}^{\mu}(x) d \mu(x) .
$$

The notation $A \approx B$ means that there is an absolute constant $c>0$, depending on $d$ and $s$ (and sometimes on other fixed parameters), such that $c^{-1} A \leq B \leq c A$.

We remark that for integer $0<s<d$ the estimate (1.2) does not hold; just let $\mu=\left.\mathcal{H}^{s}\right|_{V}$, the restriction of the $s$-dimensional Hausdorff measure to any affine $s$-plane $V$. This is connected to the well known theorem of Marstrand [M], which asserts that for $s>0$, given a Radon measure $\mu$ on $\mathbb{R}^{d}$ such that the density $\lim _{r \rightarrow 0} \theta_{\mu}^{s}(B(x, r))$ exists and is positive and finite in a set of positive $\mu$ measure, $s$ must be an integer.

In the context of integer $s$, there are also results relating rectifiability and the kind of square functions appearing in the left hand side of $(1.2)$. In [TTo it is shown that, for Radon measures $\mu$ in $\mathbb{R}^{d}$ with $\mu$-almost everywhere positive and finite lower and upper $s$-dimensional densities ( $s \in \mathbb{N}$ here) the fact that $\mu$ is $s$-rectifiable is equivalent to the $\mu$-almost everywhere finiteness of $\int_{0}^{1} \Delta_{\mu}^{s}(x, r)^{2} \frac{d r}{r}$ and also to the fact that $\lim _{r \rightarrow 0} \Delta_{\mu}^{s}(x, r)=0 \mu$-almost everywhere. It is worth also saying that the first just mentioned equivalence from [TTo is a pointwise version of a previous result in [CGLT], which characterizes the so called uniform rectifiability. In fact, in [CGLT, Lemma 3.1] a blow up argument is used, which turns to be one of the main ingredients in the proof of Theorem 1.1 (see Lemma 2.5. 
Let us remark that a suitable $p$-th version of Theorem 1.1 holds for $p \in[1, \infty)$. Indeed, almost the same proof yields that, for $0<s<d$ non-integer and such $p$,

$$
\iint_{0}^{\infty}\left|\Delta_{\mu}^{s}(x, r)\right|^{p} \frac{d r}{r} d \mu(x) \approx \iint_{0}^{\infty}\left(\frac{\mu(B(x, r))}{r^{s}}\right)^{p} \frac{d r}{r} d \mu(x),
$$

with the comparability constant depending only on $s, d$ and $p$. Notice that $\int_{0}^{\infty}\left(\frac{\mu(B(x, r))}{r^{s}}\right)^{p} \frac{d r}{r}$ coincides with the Wolff potential $\dot{W}_{\frac{p}{p+1}(d-s), p}^{\mu}(x)$, so that

$$
\iint_{0}^{\infty}\left|\Delta_{\mu}^{s}(x, r)\right|^{p} \frac{d r}{r} d \mu(x) \approx \int \dot{W}_{\frac{p}{p+1}(d-s), p}^{\mu}(x) d \mu(x) .
$$

Nevertheless, we think that the case $p=2$ is by far the most important one because of the connection with rectifiability mentioned above and because of the relationship with Riesz transforms.

In fact, Theorem 1.1 answers a question of F. Nazarov (private communication), motivated by an open problem concerning the comparability between the Wolff energy of a measure $\mu$ in $\mathbb{R}^{d}$ and the squared $L^{2}(\mu)$-norm of the $s$-Riesz transform with respect to $\mu$, for non-integer $0<s<d$. To state the problem in detail, we need to introduce some additional notation and background. For $0<s<d$, consider the signed vector valued Riesz kernels

$$
K^{s}(x)=\frac{x}{|x|^{1+s}}, x \in \mathbb{R}^{d}, x \neq 0 .
$$

The $s$-Riesz transform of a real Radon measure $\mu$ with compact support is

$$
R^{s} \mu(x)=\int K^{s}(y-x) d \mu(y)
$$

whenever the integral makes sense. To avoid delicate problems with convergence, one considers the truncated $s$-Riesz transform of $\mu$, which is defined as

$$
R_{\varepsilon}^{s} \mu(x)=\int_{|y-x|>\varepsilon} K^{s}(y-x) d \mu(y), x \in \mathbb{R}^{d}, \varepsilon>0 .
$$

One says that $R^{s} \mu$ is bounded in $L^{2}(\mu)$ if the truncated Riesz transforms $R_{\varepsilon}^{s} \mu$ are bounded in $L^{2}(\mu)$ uniformly in $\varepsilon$.

It was shown in [MPV] that given a finite Radon measure $\mu$ in $\mathbb{R}^{d}$ with growth $s$, $0<s<1$, that is, $\mu$ satisfying $\mu(B(x, r)) \leq c_{\mu} r^{s}$ for all $x \in \mathbb{R}^{d}, r>0$ and some constant $c_{\mu}>0$, one has

$$
\int \dot{W}_{\frac{2}{3}(d-s), \frac{3}{2}}^{\mu}(x) d \mu(x) \approx \sup _{\varepsilon>0} \int\left|R_{\varepsilon}^{s} \mu(x)\right|^{2} d \mu(x), \quad 0<s<1 .
$$

It is known that for the positive integers $s$ this comparability is false, while for non-integer $s \in(1, d)$ it is an open problem to prove (or disprove) it. There are some (very) partial results in this direction. In [ENV] it is shown that for $s \in(0, d)$, the Wolff energy controls the $L^{2}$-norm of the $s$-Riesz transform; in [JNV] it is proved that for $s \in(d-1, d), d \geq 2$, boundedness of the $s$-Riesz transform of $\mu$ implies $\mu$-almost everywhere finiteness of a non-linear potential of exponential type. In the special case of measures supported on Cantor type sets, the comparability $(1.3)$ has been proven for all $0<s<d$ (see [EV1], [T2 and [RT]). Since the square function on the left hand side of $(1.2)$ has a cancellative nature while the Wolff potential does not, one could think of Theorem 1.1 as being, in a 
sense, an intermediate stage towards the proof of $(1.3)$ for non-integer $1<s<d$, since the $s$-Riesz transform also has an analogous cancellative nature.

The plan of the paper is the following. In Section 2 we prove Theorem 1.1. Section 3 is devoted to the study of the relation between the $L^{2}$-norm of the $s$-Riesz transform, the Wolff energy and the square function on the left hand side of $(1.2)$, for $0<s<1$. In the final section we construct a measure with linear growth and infinite Wolff energy for which the $L^{2}(\mu)$-norm of the 1-Riesz transform with respect to $\mu$ is finite and much bigger than $\iint_{0}^{\infty} \Delta_{\mu}^{1}(x, r)^{2} \frac{d r}{r} d \mu(x)$.

Throughout the paper, the letters $c, C$ will stand for absolute constants (which may depend on $d$ and $s$ ) that may change at different occurrences.

Acknowledgments. We extend our sincere thanks to the referees for several useful comments which improved the readability of our paper.

\section{Densities and Wolff potentials}

The aim of this section is to prove our main result, Theorem 1.1. Its proof follows easily once we have at our disposal the following proposition.

Proposition 2.1. Let $s$ be positive and non-integer. Then there exists some $\delta \in(0,1)$ such that for every Radon measure $\mu$ on $\mathbb{R}^{d}$ and every open ball $B_{0} \subset \mathbb{R}^{d}$ of radius $r_{0}$,

$$
\int_{\delta r_{0}}^{\delta^{-1} r_{0}} \int_{\delta^{-1} B_{0}} \Delta_{\mu}^{s}(x, r)^{2} d \mu(x) \frac{d r}{r} \geq c(\delta) \theta_{\mu}^{s}\left(B_{0}\right)^{2} \mu\left(B_{0}\right),
$$

for some constant $c(\delta)$.

Proof of Theorem 1.1. It is enough to prove that

$$
\iint_{0}^{\infty} \Delta_{\mu}^{s}(x, r)^{2} \frac{d r}{r} d \mu(x) \gtrsim \int \dot{W}_{\frac{2}{3}(d-s), \frac{3}{2}}^{\mu}(x) d \mu(x),
$$

since the remaining inequality is immediate.

Let $\mathcal{D}$ denote the usual lattice of dyadic cubes of $\mathbb{R}^{d}$, and let $\mathcal{D}_{k} \subset \mathcal{D}, k \in \mathbb{Z}$, be the subfamily of the dyadic cubes with side length $\ell(Q)=2^{k}$. For $Q \in \mathcal{D}$, let $B_{Q}=$ $B\left(x_{Q}, r(Q)\right)$ where $x_{Q}$ is the center of $Q$ and $r(Q)=(2+\sqrt{d}) \ell(Q)$. Using Fubini's theorem and Proposition 2.1 one easily sees that

$$
\begin{aligned}
\int \dot{W}_{\frac{2}{3}(d-s), \frac{3}{2}}^{\mu}(x) d \mu(x) & =\sum_{k \in \mathbb{Z}} \sum_{Q \in \mathcal{D}_{k}} \int_{Q} \int_{2^{k}}^{2^{k+1}}\left(\frac{\mu(B(x, r))}{r^{s}}\right)^{2} \frac{d r}{r} d \mu(x) \\
& \lesssim \sum_{Q \in \mathcal{D}} \theta_{\mu}^{s}\left(B_{Q}\right)^{2} \mu\left(B_{Q}\right) \\
& \lesssim \sum_{Q \in \mathcal{D}} \int_{\delta r(Q)}^{\delta^{-1} r(Q)} \int_{\delta^{-1} B_{Q}} \Delta_{\mu}^{s}(x, r)^{2} d \mu(x) \frac{d r}{r}
\end{aligned}
$$


for some $\delta \in(0,1)$. Given $k \in \mathbb{Z}$ the family of balls $\left\{\delta^{-1} B_{Q}\right\}_{Q \in \mathcal{D}_{k}}$ has finite overlap (which depends only on $\delta$ and on the ambient dimension $d$ ). Therefore using (2.2) we get,

$$
\begin{aligned}
\int \dot{W}_{\frac{2}{3}(d-s), \frac{3}{2}}^{\mu}(x) d \mu(x) & \lesssim \sum_{k \in \mathbb{Z}} \sum_{Q \in \mathcal{D}_{k}} \int_{\delta^{-1} B_{Q}} \int_{\delta(2+\sqrt{d}) 2^{k}}^{\delta^{-1}(2+\sqrt{d}) 2^{k}} \Delta_{\mu}^{s}(x, r)^{2} \frac{d r}{r} d \mu(x) \\
& \lesssim \int \sum_{k \in \mathbb{Z}} \int_{\delta(2+\sqrt{d}) 2^{k}}^{\delta^{-1}(2+\sqrt{d}) 2^{k}} \Delta_{\mu}^{s}(x, r)^{2} \frac{d r}{r} d \mu(x) \\
& \lesssim \iint_{0}^{\infty} \Delta_{\mu}^{s}(x, r)^{2} \frac{d r}{r} d \mu(x)
\end{aligned}
$$

Before providing the proof of Proposition 2.1 we need some auxiliary results and additional notation. For any Borel function $\varphi:[0, \infty) \rightarrow \mathbb{R}$ let

$$
\varphi_{t}(x)=\frac{1}{t^{s}} \varphi\left(\frac{x}{t}\right), t>0
$$

and define

$$
\Delta_{\mu, \varphi}^{s}(x, t):=\int\left(\varphi_{t}(|y-x|)-\varphi_{2 t}(|y-x|)\right) d \mu(y),
$$

whenever the integral makes sense.

Lemma 2.2. Let $\varphi:[0, \infty) \rightarrow \mathbb{R}$ be a $\mathcal{C}^{\infty}$ function supported in $[0,2]$ which is constant in $[0,1 / 2]$. Let $x \in \mathbb{R}^{d}$ and $0 \leq r_{1}<r_{2}$. Then

$$
\int_{r_{1}}^{r_{2}}\left|\Delta_{\mu, \varphi}^{s}(x, r)\right| \frac{d r}{r} \leq c \int_{r_{1} / 2}^{2 r_{2}}\left|\Delta_{\mu}^{s}(x, r)\right| \frac{d r}{r},
$$

where $c$ depends only on $\varphi$.

Proof. This follows by writing $\varphi$ as a suitable convex combination of functions of the form $\chi_{[0, r]}$. For completeness we show the details. For $t \geq 0$ and $R>0$, we write

$$
\frac{1}{R^{s}} \varphi\left(\frac{t}{R}\right)=-\int_{0}^{\infty} \frac{1}{R^{s+1}} \varphi^{\prime}\left(\frac{r}{R}\right) \chi_{[0, r]}(t) d r
$$

so that, by Fubini and changing variables,

$$
\begin{aligned}
\Delta_{\mu, \varphi}^{s}(x, R)= & -\int_{0}^{\infty} \frac{1}{R^{s+1}} \varphi^{\prime}\left(\frac{r}{R}\right) \chi_{[0, r]}(|\cdot|) * \mu(x) d r \\
& \quad+\int_{0}^{\infty} \frac{1}{(2 R)^{s+1}} \varphi^{\prime}\left(\frac{r}{2 R}\right) \chi_{[0, r]}(|\cdot|) * \mu(x) d r \\
= & -\int_{0}^{\infty} \varphi^{\prime}(t)\left(\frac{1}{R^{s}} \chi_{[0, t R]}(|\cdot|) * \mu(x)-\frac{1}{(2 R)^{s}} \chi_{[0,2 t R]}(|\cdot|) * \mu(x)\right) d t \\
= & -\int_{1 / 2}^{2} t^{s} \varphi^{\prime}(t) \Delta_{\mu}^{s}(x, t R) d t,
\end{aligned}
$$


taking into account that $\varphi^{\prime}$ is supported on $[1 / 2,2]$ in the last identity. As a consequence we get

$$
\left|\Delta_{\mu, \varphi}^{s}(x, r)\right| \leq\left|\int_{1 / 2}^{2} t^{s} \varphi^{\prime}(t) \Delta_{\mu}^{s}(x, t r) d t\right| \lesssim \int_{1 / 2}^{2}\left|\Delta_{\mu}^{s}(x, t r)\right| d t=\int_{r / 2}^{2 r}\left|\Delta_{\mu}^{s}(x, u)\right| \frac{d u}{r} .
$$

Thus

$$
\int_{r_{1}}^{r_{2}}\left|\Delta_{\mu, \varphi}^{s}(x, r)\right| \frac{d r}{r} \lesssim \int_{r_{1}}^{r_{2}} \int_{r / 2}^{2 r}\left|\Delta_{\mu}^{s}(x, u)\right| d u \frac{d r}{r^{2}} \lesssim \int_{r_{1} / 2}^{2 r_{2}}\left|\Delta_{\mu}^{s}(x, u)\right| \frac{d u}{u}
$$

Remark 2.3. Notice that if $\varphi:[0, \infty) \rightarrow \mathbb{R}$ is a smooth function vanishing at infinity, then as in 2.3 we get

$$
\Delta_{\mu, \varphi}^{s}(x, R)=-\int_{0}^{\infty} t^{s} \varphi^{\prime}(t) \Delta_{\mu}^{s}(x, t R) d t .
$$

Lemma 2.4. Let $s$ be positive and non-integer and let $\mu$ be a non-zero Radon measure in $\mathbb{R}^{d}$. Then $\Delta_{\mu}^{s}\left(x_{0}, r_{0}\right) \neq 0$ for some $x_{0} \in \operatorname{supp}(\mu)$ and $r_{0}>0$.

Proof. By way of contradiction suppose that $\Delta_{\mu}^{s}(x, r)=0$ for all $x \in \operatorname{supp}(\mu)$ and all $r>0$. We will first show that in that case the measure $\mu$ is $s$-AD regular and we will then proceed as in the proof of [CGLT, Lemma 3.9]. Recall that $\mu$ is called $s$-Ahlfors-David regular, or $s$-AD regular, if for some constant $c_{\mu}>0$,

$$
c_{\mu}^{-1} r^{s} \leq \mu(B(x, r)) \leq c_{\mu} r^{s} \quad \text { for all } x \in \operatorname{supp}(\mu), 0<r \leq \operatorname{diam}(\operatorname{supp}(\mu)) .
$$

To prove the $s$-AD-regularity of $\mu$, assume for simplicity that $0 \in \operatorname{supp} \mu$. Since $\Delta_{\mu}^{s}(0, r)=0$ for all $r>0$, we deduce that $\mu\left(B\left(0,2^{n}\right)\right)=2^{n s} \mu(B(0,1))$ for all $n \geq 1$. For $x \in \operatorname{supp}(\mu) \cap B\left(0,2^{n-1}\right)$ and any integer $m \leq n$, using now that $\Delta_{\mu}^{s}(x, r)=0$ for all $r>0$, we infer that $\mu\left(B\left(x, 2^{m}\right)\right)=2^{(m-n) s} \mu\left(B\left(x, 2^{n}\right)\right)$. Since $B\left(0,2^{n-1}\right) \subset B\left(x, 2^{n}\right) \subset$ $B\left(0,2^{n+1}\right)$, we have

$$
2^{(n-1) s} \mu(B(0,1)) \leq \mu\left(B\left(x, 2^{n}\right)\right) \leq 2^{(n+1) s} \mu(B(0,1)) .
$$

Thus

$$
c_{0} 2^{(m-1) s} \leq \mu\left(B\left(x, 2^{m}\right)\right) \leq c_{0} 2^{(m+1) s},
$$

with $c_{0}=\mu(B(0,1))$. Since $n$ can be taken arbitrarily large and the preceding estimate holds for all $m \leq n$, the $s-\mathrm{AD}$ regularity of $\mu$ follows.

Let $\varphi(u)=e^{-u^{2}}, u \geq 0$. Then by Remark 2.3 it follows that $\Delta_{\mu, \varphi}^{s}(x, r)=0$ for all $x \in \operatorname{supp}(\mu)$ and for all $r>0$. This is equivalent to

$$
\phi_{r} * \mu(x)-\phi_{2 r} * \mu(x)=0
$$

for all $x \in \operatorname{supp}(\mu)$ and for all $r>0$, where $\phi: \mathbb{R}^{d} \rightarrow \mathbb{R}$ is defined by $\phi(y)=e^{-|y|^{2}}$. In particular

$$
\phi_{2^{-k}} * \mu(x)-\phi_{2^{k}} * \mu(x)=0 \quad \text { for all } k>0 \text { and all } x \in \operatorname{supp}(\mu) .
$$

Now consider the function $F: \mathbb{R}^{d} \rightarrow \mathbb{R}$ given by

$$
F(x)=\sum_{k>0} 2^{-k}\left(\phi_{2^{-k}} * \mu(x)-\phi_{2^{k}} * \mu(x)\right)^{2} .
$$


Taking into account that $\left|\phi_{2^{-k}} * \mu(x)-\phi_{2^{k}} * \mu(x)\right| \leq c$ for all $x \in \mathbb{R}^{d}$ and $k \in \mathbb{N}$, it is clear that $F(x)<\infty$ for all $x \in \mathbb{R}^{d}$, and so $F$ is well defined. Moreover, by 2.4 we have $F=0$ on $\operatorname{supp}(\mu)$.

Now we claim that $F(x)>0$ for all $x \in \mathbb{R}^{d} \backslash \operatorname{supp}(\mu)$. Indeed, it follows easily that

$$
\lim _{k \rightarrow \infty} \phi_{2^{-k}} * \mu(x)=0 \quad \text { for all } x \in \mathbb{R}^{d} \backslash \operatorname{supp}(\mu),
$$

while, by the $s$-AD-regularity of $\mu$,

$$
\liminf _{k \rightarrow \infty} \phi_{2^{k}} * \mu(x) \geq c c_{0} \quad \text { for all } x \in \mathbb{R}^{d} .
$$

Thus if $x \in \mathbb{R}^{d} \backslash \operatorname{supp}(\mu)$ we have $\phi_{2^{-k}} * \mu(x)-\phi_{2^{k}} * \mu(x) \neq 0$ for all large enough $k>0$, which implies that $F(x)>0$ and proves our claim. We have thus shown that $\operatorname{supp}(\mu)=F^{-1}(0)$.

Next we will prove that the zero set of $F$ is a real analytic variety. It is enough to check that $\phi_{2^{-k}} * \mu-\phi_{2^{k}} * \mu$ is a real analytic function for each $k>0$, because the zero set of a real analytic function is a real analytic variety and the intersection of any family of real analytic varieties is again a real analytic variety; see $\mathrm{Na}$. So it is enough to show that $\phi_{r} * \mu$ is a real analytic function for every $r>0$. To this end, we consider the function $f: \mathbb{C}^{d} \rightarrow \mathbb{C}$ defined by

$$
f\left(z_{1}, \ldots, z_{d}\right)=\frac{1}{r^{n}} \int \exp \left(-r^{-2} \sum_{i=1}^{d}\left(y_{i}-z_{i}\right)^{2}\right) d \mu(y) .
$$

It is easy to check that $f$ is well defined and holomorphic in the whole $\mathbb{C}^{d}$, and thus $\phi_{r} * \mu=\left.f\right|_{\mathbb{R}^{d}}$ is real analytic.

Therefore we have shown that $\operatorname{supp}(\mu)$ is an analytic variety, in particular this implies that $\operatorname{supp}(\mu)$ has Hausdorff dimension $n$ for some $n \in \mathbb{N}$. Since $\mu$ is $s$-AD regular, $\operatorname{supp}(\mu)$ has non-integer Hausdorff dimension and we have thus reached a contradiction.

The following blow-up lemma is essential for the proof of Proposition 2.1. The proof is inspired by the proof of [CGLT, Lemma 3.1]

Lemma 2.5. Let $s$ be a positive and non-integer real number. There exists some $\delta>0$ such that for every Radon measure $\mu$ in $\mathbb{R}^{d}$ which satisfies $1 \leq \mu(\bar{B}(0,1)) \leq \mu(B(0,2)) \leq$ $2^{5 s+2}$, the following estimate holds

$$
\int_{\delta}^{\delta^{-1}} \int_{x \in B\left(0, \delta^{-1}\right)}\left|\Delta_{\mu}^{s}(x, r)\right| d \mu(x) \frac{d r}{r} \geq \delta^{1 / 2} .
$$

Proof. By way of contradiction suppose that for each $m \geq 1$ there exists a Radon measure $\mu_{m}$ such that $1 \leq \mu_{m}(\bar{B}(0,1)) \leq \mu_{m}(B(0,2)) \leq 2^{5 s+2}$ which satisfies

$$
\int_{1 / m}^{m} \int_{x \in B(0, m)}\left|\Delta_{\mu_{m}}^{s}(x, r)\right| d \mu_{m}(x) \frac{d r}{r} \leq \frac{1}{m^{1 / 2}} .
$$

We will first show that the sequence $\left\{\mu_{m}\right\}$ has a subsequence $\left\{\mu_{m_{j}}\right\}$ which converges weakly * (i.e. when tested against compactly supported continuous functions) to a measure $\mu$. This follows from [Ma, Theorem 1.23] once we show that $\mu_{m}$ is uniformly bounded on 
compact sets. That is, for any compact $K \subset \mathbb{R}^{d}$, $\sup _{m} \mu_{m}(K)<\infty$. To prove this, for $n \geq 4,1 / 4<r<1 / 2$, and $x \in B(0,1)$, we write

$$
\begin{aligned}
\frac{\mu_{m}\left(B\left(0,2^{n-3}\right)\right)}{2^{(n+2) s}} \leq \frac{\mu_{m}\left(B\left(x, 2^{n} r\right)\right)}{\left(2^{n} r\right)^{s}} & \leq \sum_{k=1}^{n}\left|\Delta_{\mu_{m}}^{s}\left(x, 2^{k-1} r\right)\right|+\frac{\mu_{m}(B(x, r))}{r^{s}} \\
& \leq \sum_{k=1}^{n}\left|\Delta_{\mu_{m}}^{s}\left(x, 2^{k-1} r\right)\right|+4^{s} \mu_{m}(B(0,2)) .
\end{aligned}
$$

Integrating this estimate with respect to $\mu_{m}$ on $B(0,1)$ and with respect to $r \in[1 / 4,1 / 2]$, using 2.5 for $m$ big enough we obtain

$$
\begin{aligned}
\mu_{m}\left(B\left(0,2^{n-3}\right)\right) & \leq 2^{(n+2) s}\left[\sum_{k=1}^{n} \frac{1}{\log 2} \int_{1 / 4}^{1 / 2} \int_{B(0,1)}\left|\Delta_{\mu_{m}}^{s}\left(x, 2^{k-1} r\right)\right| d \mu_{m}(x) \frac{d r}{r}+4^{s} \mu_{m}(B(0,2))\right] \\
& \leq c(n),
\end{aligned}
$$

which proves the uniform boundedness of $\mu_{m}$ on compact sets.

Our next objective consists in proving that that $\Delta_{\mu}^{s}(x, r)=0$ for all $x \in \operatorname{supp}(\mu)$ and all $r>0$. Once this is done, the lemma would follow from Lemma 2.4 since it is easy to check that $\mu(\bar{B}(0,1)) \geq 1$, and thus $\mu$ is not identically zero.

To prove that $\Delta_{\mu}^{s}(x, r)$ vanishes identically on $\operatorname{supp} \mu$ for all $r>0$, we will show first that, given any $\mathcal{C}^{\infty}$ function $\varphi:[0, \infty) \rightarrow \mathbb{R}$ which is supported in $[0,2]$ and constant in $[0,1 / 2]$, we have

$$
\int_{0}^{\infty} \int_{x \in \mathbb{R}^{d}}\left|\Delta_{\mu, \varphi}^{s}(x, r)\right| d \mu(x) \frac{d r}{r}=0 .
$$

The proof of this fact is elementary. Fix $m_{0}$ and let $\eta>0$. Set $K=\left[2 / m_{0}, m_{0} / 2\right] \times$ $\bar{B}\left(0, m_{0}\right)$. Now $\left\{y \rightarrow \varphi_{t}(|x-y|)-\varphi_{2 t}(|x-y|),(t, x) \in K\right\}$ is an equicontinuous family of continuous functions supported inside a fixed compact set. Hence setting, $\phi(x)=$ $\varphi(|x|), x \in \mathbb{R}^{d}$, we get that $\left(\phi_{t}-\phi_{2 t}\right) * \mu_{m_{j}}(x)$ converges to $\left(\phi_{t}-\phi_{2 t}\right) * \mu(x)$ uniformly on $K$. It therefore follows that

$$
\begin{aligned}
\iint_{K}\left|\Delta_{\mu, \varphi}^{s}(x, t)\right| d \mu(x) \frac{d t}{t} & =\iint_{K}\left|\left(\phi_{t}-\phi_{2 t}\right) * \mu(x)\right| d \mu(x) \frac{d t}{t} \\
& =\lim _{j} \int_{2 / m_{0}}^{m_{0} / 2} \int_{x \in \bar{B}\left(0, m_{0}\right)}\left|\left(\phi_{t}-\phi_{2 t}\right) * \mu_{m_{j}}(x)\right| d \mu_{m_{j}}(x) \frac{d t}{t} \\
& =\lim _{j} \int_{x \in \bar{B}\left(0, m_{0}\right)} \int_{2 / m_{0}}^{m_{0} / 2}\left|\Delta_{\mu_{m_{j}}, \varphi}^{s}(x, t)\right| \frac{d t}{t} d \mu_{m_{j}}(x) \\
& \lesssim \lim _{j} \int_{x \in \bar{B}\left(0, m_{0}\right)} \int_{1 / m_{0}}^{m_{0}}\left|\Delta_{\mu_{m_{j}}}^{s}(x, t)\right| d \mu_{m_{j}}(x) \frac{d t}{t}=0
\end{aligned}
$$

by Lemma 2.2 and $(2.5)$. Since this holds for any $m_{0} \geq 1$, our claim 2.6 is proved.

Denote by $G$ the subset of those points $x \in \operatorname{supp}(\mu)$ such that

$$
\int_{0}^{\infty}\left|\Delta_{\mu, \varphi}^{s}(x, r)\right| \frac{d r}{r}=0
$$

It is clear now that $G$ has full $\mu$-measure. By continuity, it follows that $\Delta_{\mu, \varphi}^{s}(x, r)=0$ for all $x \in \operatorname{supp} \mu$ and all $r>0$. Finally, by taking a suitable sequence of $\mathcal{C}^{\infty}$ functions 
$\varphi_{k}$ which converge to $\chi_{[0,1]}$ we infer that $\Delta_{\mu}^{s}(x, r)=0$ for all $x \in \operatorname{supp} \mu$ and $r>0$. By Lemma 2.4, this is impossible.

By renormalizing the preceding lemma we get:

Lemma 2.6. Let $s$ be a positive and non-integer real number. There exists some $\delta>0$ such that for every Radon measure $\mu$ in $\mathbb{R}^{d}$ and every open ball of radius $r_{0}$ such that $0<\mu\left(\bar{B}_{0}\right) \leq \mu\left(2 B_{0}\right) \leq 2^{5 s+2} \mu\left(\bar{B}_{0}\right)$, the following estimate holds

$$
\int_{\delta r_{0}}^{\delta^{-1} r_{0}} \int_{x \in \delta^{-1} B_{0}}\left|\Delta_{\mu}^{s}(x, r)\right| d \mu(x) \frac{d r}{r} \geq \delta^{1 / 2} \frac{\mu\left(\bar{B}_{0}\right)^{2}}{r_{0}^{s}} .
$$

Proof. Let $T: \mathbb{R}^{d} \rightarrow \mathbb{R}^{d}$ be an affine transformation which maps $\bar{B}_{0}$ to $\bar{B}(0,1)$. Consider the measure $\sigma=\frac{1}{\mu\left(\bar{B}_{0}\right)} T_{\#} \mu$, where as usual $T_{\#} \mu(E):=\mu\left(T^{-1}(E)\right)$, and apply the preceding lemma to $\sigma$.

We can now complete the proof of Proposition 2.1.

Proof of Proposition 2.1. Let $B_{0}$ be an open ball of radius $r_{0}$ such that $\mu\left(B_{0}\right)>0$. Let $\delta \in(0,1)$ to be fixed below and let $k=k(\delta)$ be such that $2^{-k} \leq \delta<2^{-k+1}$. If

$$
\int_{2^{-k-2} r_{0}}^{2^{k+2} r_{0}} \int_{2^{k+2} B_{0}} \Delta_{\mu}^{s}(x, r)^{2} d \mu(x) \frac{d r}{r}>\delta^{4} \theta_{\mu}^{s}\left(B_{0}\right)^{2} \mu\left(B_{0}\right)
$$

we are done. Otherwise, there exists some $x \in B_{0}$ such that

$$
\int_{2^{-k-2} r_{0}}^{2^{k+2} r_{0}} \Delta_{\mu}^{s}(x, r)^{2} \frac{d r}{r} \leq 2 \delta^{4} \theta_{\mu}^{s}\left(B_{0}\right)^{2} .
$$

Notice also that after changing variables, for any $n \in \mathbb{Z}$, we have

$$
\int_{r_{0} / 2}^{r_{0}} \Delta_{\mu}^{s}\left(x, 2^{n} r\right)^{2} \frac{d r}{r}=\int_{2^{n-1} r_{0}}^{2^{n} r_{0}} \Delta_{\mu}^{s}(x, r)^{2} \frac{d r}{r} .
$$

Therefore

$$
\int_{2^{-k-2} r_{0}}^{2^{k+2} r_{0}} \Delta_{\mu}^{s}(x, r)^{2} \frac{d r}{r}=\int_{r_{0} / 2}^{r_{0}} \sum_{n=-k-1}^{k+2} \Delta_{\mu}^{s}\left(x, 2^{n} r\right)^{2} \frac{d r}{r} .
$$

Using (2.7), (2.8) and applying Chebyshev's inequality with respect to the measure $d t / t$ we find some $t \in\left[r_{0} / 2, r_{0}\right]$ such that

$$
\sum_{n=-k-1}^{k+2} \Delta_{\mu}^{s}\left(x, 2^{n} t\right)^{2} \leq \frac{4 \delta^{4}}{\log 2} \theta_{\mu}^{s}\left(B_{0}\right)^{2} .
$$

In particular,

$$
\left|\Delta_{\mu}^{s}\left(x, 2^{n} t\right)\right| \leq \frac{2 \delta^{2}}{\sqrt{\log 2}} \theta_{\mu}^{s}\left(B_{0}\right)
$$

for $n=-k-1, \ldots, k+2$. This implies that

$$
\left|\frac{\mu\left(B\left(x, 2^{k+3} t\right)\right)}{\left(2^{k+3} t\right)^{s}}-\frac{\mu(B(x, t))}{t^{s}}\right| \leq 4(k+2)\left(2^{-k+1}\right)^{2} \theta_{\mu}^{s}\left(B_{0}\right) \leq \theta_{\mu}^{s}\left(B_{0}\right) .
$$


Therefore,

$$
\frac{\mu\left(2 \delta^{-1} B_{0}\right)}{\left(2 \delta^{-1} r_{0}\right)^{s}} \leq 2^{3 s} \frac{\mu\left(B\left(x, 2^{k+3} t\right)\right)}{\left(2^{k+3} t\right)^{s}} \leq 2^{3 s}\left(\frac{\mu(B(x, t))}{t^{s}}+2^{s} \theta_{\mu}^{s}\left(2 B_{0}\right)\right) \leq 2^{5 s+1} \theta_{\mu}^{s}\left(2 B_{0}\right),
$$

and so

$$
\left.\mu\left(2 \delta^{-1} B_{0}\right)\right) \leq 2^{5 s+1} \delta^{-s} \mu\left(2 B_{0}\right) .
$$

In the same way (in fact, just setting $\delta=1 / 2$ ) one easily deduces that

$$
\mu\left(4 B_{0}\right) \leq 2^{5 s+2} \mu\left(2 B_{0}\right) .
$$

Therefore we can apply Lemma 2.6 to $2 B_{0}$ and obtain

$$
\int_{2 \delta r_{0}}^{2 \delta^{-1} r_{0}} \int_{x \in 2 \delta^{-1} B_{0}}\left|\Delta_{\mu}^{s}(x, r)\right| d \mu(x) \frac{d r}{r}>\delta^{1 / 2} \frac{\mu\left(2 B_{0}\right)^{2}}{\left(2 r_{0}\right)^{s}} .
$$

By Cauchy-Schwartz and (2.10), it follows that

$$
\mu\left(2 \delta^{-1} B_{0}\right) \log \left(\delta^{-2}\right) \int_{2 \delta r_{0}}^{2 \delta^{-1} r_{0}} \int_{x \in 2 \delta^{-1} B_{0}} \Delta_{\mu}^{s}(x, r)^{2} d \mu(x) \frac{d r}{r}>\delta \frac{\mu\left(2 B_{0}\right)^{4}}{\left(2 r_{0}\right)^{2 s}} .
$$

Finally using 2.9 we have,

$$
\int_{2 \delta r_{0}}^{2 \delta^{-1} r_{0}} \int_{x \in 2 \delta^{-1} B_{0}} \Delta_{\mu}^{s}(x, r)^{2} d \mu(x) \frac{d r}{r} \gtrsim \frac{\delta^{s+1}}{\log \left(\delta^{-2}\right)} \theta_{\mu}^{s}\left(B_{0}\right)^{2} \mu\left(B_{0}\right) .
$$

\section{Relationship With the $s$-Riesz transform For $0<s<1$}

It was shown in [MPV] that for a finite Radon measure $\mu$ in $\mathbb{R}^{d}$, we have

$$
\sup _{\varepsilon>0} \int\left|R_{\varepsilon}^{s}(\mu)(x)\right|^{2} d \mu(x) \approx \int \dot{W}_{\frac{2}{3}(d-s), \frac{3}{2}}^{\mu}(x) d \mu(x) .
$$

In this section we extend this result to the case of non-finite Radon measures. In part, our motivation stems from the counterexample that we will construct in Section 4 for the case $s=1$, which consists of a non-finite Radon measure for which the squared $L^{2}(\mu)$-norm of the 1-Riesz transform of $\mu$ is not comparable to $\iint_{0}^{\infty} \Delta_{\mu}^{1}(x, r)^{2} \frac{d r}{r} d \mu(x)$.

The next proposition is stated in terms of the doubly truncated Riesz transform of $\mu$. Given $0<\varepsilon_{1}<\varepsilon_{2}$, this is defined as

$$
R_{\varepsilon_{1}, \varepsilon_{2}}^{s}(\mu)(x)=\int_{\varepsilon_{1}<|y-x| \leq \varepsilon_{2}} K^{s}(y-x) d \mu(y) .
$$

Proposition 3.1. Let $\mu$ be a Radon measure in $\mathbb{R}^{d}$ and $0<s<1$. Then the following statements hold:

(a) For every $\varepsilon_{1}, \varepsilon_{2}>0$,

$$
\int\left|R_{\varepsilon_{1}, \varepsilon_{2}}^{s}(\mu)(x)\right|^{2} d \mu(x) \leq C \int \dot{W}_{\frac{2}{3}(d-s), \frac{3}{2}}^{\mu}(x) d \mu(x),
$$

with $C$ independent of $\varepsilon_{1}$ and $\varepsilon_{2}$. 
(b) If $\mu$ is such that $\liminf _{r \rightarrow \infty} \frac{\mu(B(0, r))^{3}}{r^{2 s}}<\infty$, then

$$
\int \dot{W}_{\frac{2}{3}(d-s), \frac{3}{2}}^{\mu}(x) d \mu(x) \leq C \sup _{\varepsilon_{2}>\varepsilon_{1}>0} \int\left|R_{\varepsilon_{1}, \varepsilon_{2}}^{s}(\mu)(x)\right|^{2} d \mu(x) .
$$

Remark 3.2. First, let us mention that it is easy to see that there exist non-finite measures $\mu$ with finite Wolff energy.

Second, notice that in general (3.3) does not hold without assuming the finiteness of $\liminf _{r \rightarrow \infty} \frac{\mu(B(0, r))^{3}}{r^{2 s}}$. Take for example $\mu=\left.\mathcal{H}^{1}\right|_{\mathbb{R}}$, then by antisymmetry one has $\int\left|R_{\varepsilon_{1}, \varepsilon_{2}}^{s}(\mu)\right|^{2} d \mu=$ 0 , while $\int \dot{W}_{\frac{2}{3}(d-s), \frac{3}{2}}^{\mu} d \mu=\infty$.

On the other hand, it is easy to check that

$$
\int \dot{W}_{\frac{2}{3}(d-s), \frac{3}{2}}^{\mu}(x) d \mu(x)<\infty \quad \Rightarrow \quad \lim _{r \rightarrow \infty} \frac{\mu(B(0, r))^{3}}{r^{2 s}}=0 .
$$

By combining Proposition 3.1 and Theorem 1.1 we get the following corollary

Corollary 3.3. Let $\mu$ be a Radon measure in $\mathbb{R}^{d}$ and let $0<s<1$. If $\mu$ is such that $\liminf _{r \rightarrow \infty} \frac{\mu(B(0, r))^{3}}{r^{2 s}}<\infty$, then

$$
\sup _{\varepsilon_{1}, \varepsilon_{2}>0} \int\left|R_{\varepsilon_{1}, \varepsilon_{2}}^{s}(\mu)(x)\right|^{2} d \mu(x) \approx \int \dot{W}_{\frac{2}{3}(d-s), \frac{3}{2}}^{\mu}(x) d \mu(x) \approx \iint_{0}^{\infty} \Delta_{\mu}^{s}(x, r)^{2} \frac{d r}{r} d \mu(x) .
$$

Before proving the proposition we need to recall the definition of balls with thin boundaries. Given $t>0$, a ball $B(x, r)$ is said to have $t$-thin boundary (or just thin boundary) if

$$
\mu(\{y \in B(x, 2 r): \operatorname{dist}(y, \partial B(x, r)) \leq \lambda r\}) \leq t \lambda \mu(B(x, 2 r))
$$

for all $\lambda>0$. The following result is well known. For the proof (with cubes instead of balls) see Lemma 9.43 of [T3], for example.

Lemma 3.4. Let $\mu$ be a Radon measure on $\mathbb{R}^{d}$. Let $t$ be some constant big enough (depending only on $d$ ). Let $B(x, r) \subset \mathbb{R}^{d}$ be any fixed ball. Then there exists $r^{\prime} \in[r, 2 r]$ such that the ball $B\left(x, r^{\prime}\right)$ has t-thin boundary.

Now we turn to the proof of Proposition 3.1.

Proof of Proposition 3.1. If $\mu$ is a compactly supported Radon measure and $0<s<1$, then by [MPV], for any $r>0$,

$$
\int_{B(0, r)}\left|R_{\varepsilon_{1}}^{s}\left(\chi_{B(0, r)} \mu\right)(x)\right|^{2} d \mu(x) \lesssim \int \dot{W}_{\frac{2}{3}(d-s), \frac{3}{2}}^{\mu}(x) d \mu(x)
$$

for all $\varepsilon_{1}>0$ and $r>0$. Therefore,

$$
\int_{B\left(0, r_{0}\right)}\left|R_{\varepsilon_{1}, \varepsilon_{2}}^{s}\left(\chi_{B(0, r)} \mu\right)(x)\right|^{2} d \mu(x) \lesssim \int \dot{W}_{\frac{2}{3}(d-s), \frac{3}{2}}^{\mu}(x) d \mu(x)
$$

for any $r_{0}<r$ and $\varepsilon_{1}, \varepsilon_{2}>0$. Since

$$
\lim _{r \rightarrow \infty}\left|R_{\varepsilon_{1}, \varepsilon_{2}}^{s}\left(\chi_{B(0, r)} \mu\right)(x)\right|=\left|R_{\varepsilon_{1}, \varepsilon_{2}}^{s}(\mu)(x)\right|
$$


and for a fixed $r_{0}>0$ and $x \in B\left(0, r_{0}\right)$,

$$
\left|R_{\varepsilon_{1}, \varepsilon_{2}}^{s}(\mu)(x)\right| \leq \frac{\mu\left(B\left(0, r_{0}+\varepsilon_{2}\right)\right)}{\varepsilon_{1}^{s}}=C_{r_{0}, \varepsilon_{1}, \varepsilon_{2}},
$$

the dominated convergence theorem proves (3.2).

Now we deal with inequality (3.3). Clearly we may assume that

$$
\sup _{\varepsilon_{1}, \varepsilon_{2}} \int\left|R_{\varepsilon_{1}, \varepsilon_{2}}^{s}(\mu)(x)\right|^{2} d \mu(x)<\infty,
$$

since otherwise the statement (b) is trivial. Using [MPV], given $r>0$ and taking $\varepsilon_{2}=2 r$ we get

$$
\begin{aligned}
\int_{B(0, r)} \dot{W}_{\frac{2}{3}(d-s), \frac{3}{2}}^{\chi_{B(0, r)} \mu}(x) d \mu(x) \lesssim & \sup _{\varepsilon_{1}>0} \int_{B(0, r)}\left|R_{\varepsilon_{1}, \varepsilon_{2}}^{s}\left(\chi_{B(0, r)} \mu\right)(x)\right|^{2} d \mu(x) \\
\lesssim & \sup _{\varepsilon_{1}>0} \int\left|R_{\varepsilon_{1}, \varepsilon_{2}}^{s}(\mu)(x)\right|^{2} d \mu(x) \\
& +\sup _{\varepsilon_{1}>0} \int_{B(0, r)}\left|R_{\varepsilon_{1}, \varepsilon_{2}}^{s}\left(\chi_{B(0, r) c} \mu\right)(x)\right|^{2} d \mu(x) .
\end{aligned}
$$

We claim that if $B(0, r)$ has thin boundary, then

$$
\left|R_{\varepsilon_{1}, \varepsilon_{2}}^{s}\left(\chi_{B(0, r)^{c}} \mu\right)(x)\right| \lesssim \theta_{\mu}^{s}(B(0,3 r)) \quad \text { for } \varepsilon_{2}=2 r \text { and } x \in B(0, r) .
$$

Assuming this for the moment, we get

$$
\int_{B(0, r)}\left|R_{\varepsilon_{1}, \varepsilon_{2}}^{s}\left(\chi_{B(0, r)^{c}} \mu\right)(x)\right|^{2} d \mu(x) \lesssim \theta_{\mu}^{s}(B(0,3 r))^{2} \mu(B(0, r)),
$$

and thus

$$
\begin{aligned}
\int_{B(0, r)} & \dot{W}_{\frac{2}{3}(d-s), \frac{3}{2}}^{\chi_{B(0, r)} \mu}(x) d \mu(x) \lesssim \\
& \sup _{\varepsilon_{2}>\varepsilon_{1}>0} \int\left|R_{\varepsilon_{1}, \varepsilon_{2}}^{s}(\mu)(x)\right|^{2} d \mu(x)+\theta_{\mu}^{s}(B(0,3 r))^{2} \mu(B(0,3 r)) .
\end{aligned}
$$

By the assumption in (b), there exists a sequence $r_{k} \rightarrow \infty$ such that

$$
\sup _{k>0} \theta_{\mu}^{s}\left(B\left(0, r_{k}\right)\right)^{2} \mu\left(B\left(0, r_{k}\right)\right)<\infty .
$$

By Lemma 3.4 , for each $k$ there exists some $\widetilde{r}_{k} \in\left[\frac{1}{6} r_{k}, \frac{1}{3} r_{k}\right]$ such that the ball $B\left(0, \widetilde{r}_{k}\right)$ has thin boundary. Since $\theta_{\mu}^{s}\left(B\left(0,3 \widetilde{r}_{k}\right)\right)^{2} \mu\left(B\left(0,3 \widetilde{r}_{k}\right)\right) \lesssim \theta_{\mu}^{s}\left(B\left(0, r_{k}\right)\right)^{2} \mu\left(B\left(0, r_{k}\right)\right)$, from (3.7) we deduce

$$
\int_{B\left(0, \widetilde{r}_{k}\right)} \dot{W}_{\frac{2}{3}(d-s), \frac{3}{2}}^{\chi_{B\left(0, \widetilde{r}_{k}\right) \mu}}(x) d \mu(x) \lesssim \sup _{\varepsilon_{2}>\varepsilon_{1}>0} \int\left|R_{\varepsilon_{1}, \varepsilon_{2}}^{s}(\mu)(x)\right|^{2} d \mu(x)+\theta_{\mu}^{s}\left(B\left(0, r_{k}\right)\right)^{2} \mu\left(B\left(0, r_{k}\right)\right) .
$$

Letting $k \rightarrow \infty$, we obtain

$$
\int \dot{W}_{\frac{2}{3}(d-s), \frac{3}{2}}^{\mu}(x) d \mu(x) \lesssim \sup _{\varepsilon_{2}>\varepsilon_{1}>0} \int\left|R_{\varepsilon_{1}, \varepsilon_{2}}^{s}(\mu)(x)\right|^{2} d \mu(x)+\liminf _{r \rightarrow \infty} \frac{\mu(B(0, r))^{3}}{r^{2 s}} .
$$


By the assumptions in (b) the right hand above is finite and thus $\int \dot{W}_{\frac{2}{3}(d-s), \frac{3}{2}}^{\mu}(x) d \mu(x)<$ $\infty$, which in turn implies that $\lim _{r \rightarrow \infty} \frac{\mu(B(0, r))^{3}}{r^{2 s}}=0$ by 3.4 . Hence the statement $(\mathrm{b})$ of the proposition follows from $(3.8)$.

It remains to prove (3.6). For $x \in B(0, r)$ and $\varepsilon_{2}=2 r$, we have

$$
\begin{aligned}
\left|R_{\varepsilon_{1}, \varepsilon_{2}}^{s}\left(\chi_{B(0, r)^{c}} \mu\right)(x)\right| & \leq \int_{B(0,3 r) \backslash B(0, r)} \frac{1}{|y-x|^{s}} d \mu(y) \\
& \lesssim \sum_{k \geq 1} \frac{\mu\left(B\left(x, 2^{-k} r\right) \cap B(0,3 r) \backslash B(0, r)\right)}{\left(2^{-k} r\right)^{s}} .
\end{aligned}
$$

Note now that if $B\left(x, 2^{-k} r\right) \cap B(0,3 r) \backslash B(0, r) \neq \varnothing$, then

$$
B\left(x, 2^{-k} r\right) \cap B(0,3 r) \subset B(0,3 r) \cap U_{2^{-k+1}}(\partial B(0, r)),
$$

where $U_{\delta}(A)$ stands for the $\delta$-neighborhood of $A$. Thus, in any case we have

$$
\mu\left(B\left(x, 2^{-k} r\right) \cap B(0,3 r) \backslash B(0, r)\right) \lesssim 2^{-k} \mu(B(0,3 r)),
$$

because $B(0, r)$ has thin boundary. Therefore,

$$
\left|R_{\varepsilon_{1}, \varepsilon_{2}}^{s}\left(\chi_{B(0, r)^{c} \mu}\right)(x)\right| \lesssim \sum_{k \geq 1} 2^{-k(1-s)} \frac{\mu(B(0,3 r))}{r^{s}} \lesssim \theta_{\mu}^{s}(B(0,3 r)),
$$

as claimed.

\section{Counterexample in the integer Case $s=1$}

In this section we give an example of an infinite measure in the plane such that the quantities

$$
\int\left|R^{1}(\mu)(x)\right|^{2} d \mu(x), \quad \int W_{\frac{2}{3}, \frac{3}{2}}^{\mu}(x) d \mu(x), \quad \iint_{0}^{\infty} \Delta_{\mu}^{1}(x, r)^{2} \frac{d r}{r} d \mu(x)
$$

are not comparable, in contrast to the result stated in Corollary 3.3 for $0<s<1$. Our example consists of a measure $\mu$ with linear growth (i.e. growth 1), infinite Wolff energy, for which the squared $L^{2}(\mu)$-norm of the 1-Riesz transform with respect to $\mu$ is finite and much bigger than $\iint_{0}^{\infty} \Delta_{\mu}^{1}(x, r)^{2} \frac{d r}{r} d \mu(x)$. We think that this fact is quite surprising, because for general measures $\mu$ with linear growth (i.e., with growth 1 ) in the complex plane, it has been recently shown in [T4] that

$$
\int_{Q}\left|R_{\mu}^{1} \chi_{Q}\right|^{2} d \mu \leq C \mu(Q) \quad \text { for every square } Q \subset \mathbb{C}
$$

if and only if

$$
\int_{Q} \int_{0}^{\infty} \Delta_{\chi_{Q} \mu}^{1}(x, r)^{2} \frac{d r}{r} d \mu(x) \leq C^{\prime} \mu(Q) \quad \text { for every square } Q \subset \mathbb{C} .
$$

Now we turn to the construction of the measure for our counterexample. Consider the curve $\Gamma_{\alpha}$ as in Figure 1, for $0<\alpha \leq \pi / 4$. In particular, $\Gamma_{\alpha} \subset \mathbb{R}^{2}$ can be realized as the 


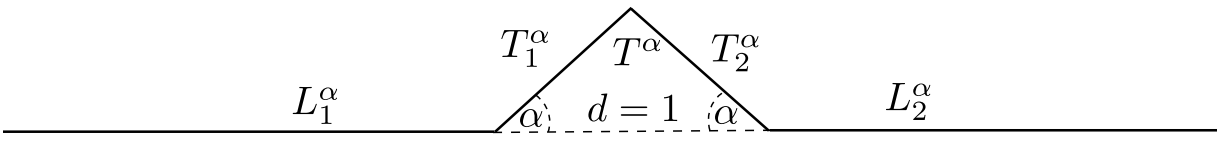

Figure 1. The curve $\Gamma_{\alpha}$.

graph of the piecewise linear function $f: \mathbb{R} \rightarrow \mathbb{R}$ defined by

$$
f(x)= \begin{cases}0 & \text { if } x \in(-\infty,-1 / 2] \cup[1 / 2, \infty), \\ \tan \alpha(x+1 / 2) & \text { if } x \in(-1 / 2,0] \\ -\tan \alpha(x-1 / 2) & \text { if } x \in(0,1 / 2)\end{cases}
$$

We set

- $L_{1}^{\alpha}=(-\infty, 1 / 2] \times\{0\}$,

- $T_{1}^{\alpha}=\left\{(x, f(x)) \in \mathbb{R}^{2}: x \in[-1 / 2,0]\right\}$,

- $T_{2}^{\alpha}=\left\{(x, f(x)) \in \mathbb{R}^{2}: x \in[0,1 / 2]\right\}$,

- $L_{2}^{\alpha}=[1 / 2, \infty) \times\{0\}$

- $T^{\alpha}=T_{1}^{\alpha} \cup T_{2}^{\alpha}$.

We will show that, for the 1-dimensional Hausdorff measure $\mu=\left.\mathcal{H}^{1}\right|_{\Gamma_{\alpha}}$, the three quantities in (4.1) are not comparable. Note that, strictly speaking, this fact cannot be consider as a counterexample to Corollary 3.3 for the case $s=1$, since the assumption $\liminf _{r \rightarrow \infty} \frac{\mu(B(0, r))^{3}}{r^{2}}<\infty$ does not hold.

Proposition 4.1. Let $\alpha \in(0, \pi / 4]$. Then

$$
\left.\iint_{0}^{\infty} \Delta_{\left.\mathcal{H}^{1}\right|_{\Gamma_{\alpha}}}^{1}(x, r)^{2} \frac{d r}{r} d \mathcal{H}^{1}\right|_{\Gamma_{\alpha}}(x) \lesssim \sin ^{4} \alpha
$$

Also,

$$
\sup _{\varepsilon_{1}, \varepsilon_{2}>0} \int\left|R_{\varepsilon_{1}, \varepsilon_{2}}^{1}(\mu)(x)\right|^{2} d \mu(x) \approx \sin ^{2} \alpha .
$$

It is clear that, letting $\alpha \rightarrow 0$, we will get

$$
\iint_{0}^{\infty} \Delta_{\left.\mathcal{H}^{1}\right|_{\Gamma_{\alpha}}}^{1}(x, r)^{2} \frac{d r}{r} \ll \sup _{\varepsilon_{1}, \varepsilon_{2}>0} \int\left|R_{\varepsilon_{1}, \varepsilon_{2}}^{1}(\mu)(x)\right|^{2} d \mu(x) .
$$

On the other hand, it is easy to check that $\left.\left.\int W_{\frac{2}{3}, \frac{3}{2}}^{\mathcal{H}^{1}}\right|_{\alpha}(x) d \mathcal{H}^{1}\right|_{\Gamma_{\alpha}}(x)=\infty$.

To prove Proposition 4.1, we consider the auxiliary 1-AD regular measure on $\Gamma_{\alpha}$ :

$$
\mu_{\alpha}=\left.\cos \alpha \mathcal{H}^{1}\right|_{T^{\alpha}}+\left.\mathcal{H}^{1}\right|_{\Gamma_{\alpha} \backslash T_{\alpha}},
$$

for which the following holds:

Lemma 4.2. Let $\alpha \in(0, \pi / 4]$. Then

$$
\iint_{0}^{\infty} \Delta_{\mu_{\alpha}}^{1}(x, r)^{2} \frac{d r}{r} d \mu_{\alpha}(x) \lesssim \sin ^{4} \alpha
$$

Using Lemma 4.2, we are now able to prove Proposition 4.1. 
Proof of Proposition 4.1. To show (4.2), notice that

$$
\begin{aligned}
\Delta_{\mathcal{H}^{1} \mid \Gamma_{\alpha}}^{1}(x, r)-\Delta_{\mu_{\alpha}}^{1}(x, r) & \leq c(1-\cos \alpha) \frac{\mathcal{H}^{1}\left(T^{\alpha} \cap B(x, 2 r)\right)}{r} \\
& \leq c \alpha^{2} \frac{\mathcal{H}^{1}\left(T^{\alpha} \cap B(x, 2 r)\right)}{r}
\end{aligned}
$$

Hence,

$$
\begin{aligned}
\left(\int_{1 / 10}^{\infty} \int \Delta_{\mathcal{H}^{1} \mid \Gamma_{\alpha}}^{1}(x, r)^{2}\right. & \left.\left.d \mathcal{H}^{1}\right|_{\Gamma_{\alpha}}(x) \frac{d r}{r}\right)^{1 / 2} \\
\lesssim & \left(\left.\int_{1 / 10}^{\infty} \int\left(\Delta_{\left.\mathcal{H}^{1}\right|_{\Gamma_{\alpha}}}^{1}(x, r)-\Delta_{\mu_{\alpha}}^{1}(x, r)\right)^{2} d \mathcal{H}^{1}\right|_{\Gamma_{\alpha}}(x) \frac{d r}{r}\right)^{1 / 2} \\
& +\left(\left.\int_{1 / 10}^{\infty} \int \Delta_{\mu_{\alpha}}^{1}(x, r)^{2} d \mathcal{H}^{1}\right|_{\Gamma_{\alpha}}(x) \frac{d r}{r}\right)^{1 / 2}=A+B
\end{aligned}
$$

the last identity being a definition for $A$ and $B$. By Lemma 4.2 , we have $B \leq c \alpha^{2}$. Concerning $A$, using (4.4) and the linear growth of $\left.\mathcal{H}^{1}\right|_{\Gamma_{\alpha}}$, we get

$$
\begin{aligned}
A^{2} & \leq\left.\alpha^{4} \int_{r \geq 1 / 10} \int_{|x| \leq 10 r} \frac{\mathcal{H}^{1}\left(T^{\alpha} \cap B(x, 2 r)\right)^{2}}{r^{2}} d \mathcal{H}^{1}\right|_{\Gamma_{\alpha}}(x) \frac{d r}{r} \\
& \leq \alpha^{4} \int_{r \geq 1 / 10} \int_{|x| \leq 10 r} \frac{\left.d \mathcal{H}^{1}\right|_{\Gamma_{\alpha}}(x)}{r^{3}} d r \leq c \alpha^{4} \int_{r \geq 10} \frac{d r}{r^{2}} \leq c \alpha^{4} .
\end{aligned}
$$

Now we write

$$
\begin{aligned}
\left(\int_{0}^{1 / 10} \int \Delta_{\left.\mathcal{H}^{1}\right|_{\Gamma}}^{1}(x, r)^{2}\right. & \left.\left.d \mathcal{H}^{1}\right|_{\Gamma_{\alpha}}(x) \frac{d r}{r}\right)^{1 / 2} \\
\lesssim & \left(\left.\int_{0}^{1 / 10} \int\left(\Delta_{\left.\mathcal{H}^{1}\right|_{\Gamma_{\alpha}}}^{1}(x, r)-\Delta_{\mu_{\alpha}}^{1}(x, r)\right)^{2} d \mathcal{H}^{1}\right|_{\Gamma_{\alpha}}(x) \frac{d r}{r}\right)^{1 / 2} \\
& +\left(\left.\int_{0}^{1 / 10} \int \Delta_{\mu_{\alpha}}^{1}(x, r)^{2} d \mathcal{H}^{1}\right|_{\Gamma_{\alpha}}(x) \frac{d r}{r}\right)^{1 / 2}=C+D
\end{aligned}
$$

the last identity being the definition of $C$ and $D$. Again by Lemma $4.2, D \leq c \alpha^{2}$. To estimate $C$, we consider the vertices $\left\{z_{a}\right\}=L_{1}^{\alpha} \cap T_{1}^{\alpha},\left\{z_{b}\right\}=T_{1}^{\alpha} \cap T_{2}^{\alpha}$ and $\left\{z_{c}\right\}=T_{2}^{\alpha} \cap L_{2}^{\alpha}$. It is easy to check that $\Delta_{\left.\mathcal{H}^{1}\right|_{\Gamma_{\alpha}}}^{1}(x, r)-\Delta_{\mu_{\alpha}}^{1}(x, r)$ vanishes unless $z_{a} \in B(x, 2 r), z_{b} \in B(x, 2 r)$ or $z_{c} \in B(x, 2 r)$. Then we split the integral in $C$ into three integrals according to the three preceding cases. Since they are treated similarly, we will deal only with the first one. 
Using again 4.4, we get

$$
\begin{aligned}
\left.\int_{0}^{1 / 10} \int_{z_{a} \in B(x, 2 r)} \Delta_{\left.\mathcal{H}^{1}\right|_{\Gamma_{\alpha}}}^{1}(x, r)^{2} d \mathcal{H}^{1}\right|_{\Gamma_{\alpha}}(x) \frac{d r}{r} \\
\\
\left.\lesssim \alpha^{4} \int_{0}^{1 / 10} \int_{\left|x-z_{a}\right|<2 r}\left(\frac{\mathcal{H}^{1}\left(T^{\alpha} \cap B(x, 2 r)\right)}{r}\right)^{2} d \mathcal{H}^{1}\right|_{\Gamma_{\alpha}}(x) \frac{d r}{r} \leq c \alpha^{4} .
\end{aligned}
$$

Gathering all the preceding estimates, 4.2 follows.

The proof of (4.3) can be obtained either by [T1, Corollary 1.4] or by more elementary methods (which we leave for the reader).

Proof of Lemma 4.2. We fix $\alpha \in(0, \pi / 4]$. To simplify notation write $\mu, \Gamma, T, L$ and so on, instead of $\mu_{\alpha}, \Gamma_{\alpha}, T^{\alpha}, L_{\alpha}$.

To estimate $\Delta_{\mu}^{1}(x, r)$ for $x \in \Gamma$ and $r>0$, note first that $\Delta_{\mu}(x, r)$ vanishes if one of the following conditions holds:

- $B(x, 2 r) \cap \Gamma \subset L_{i}$ for $i=1$ or 2 ,

- $B(x, 2 r) \cap \Gamma \subset T_{i}$ for $i=1$ or 2 .

- $x \in L_{1} \cup L_{2}$ and $T \subset B(x, r)$.

In this case, we set $(x, r) \in Z$.

In the case $(x, r) \notin Z$, we write

$$
\begin{aligned}
\left|\Delta_{\mu}^{1}(x, r)\right| & \leq\left|\frac{\mu(B(x, r))-2 r}{r}\right|+\left|\frac{\mu(B(x, 2 r))-4 r}{2 r}\right| \\
& =: \delta_{\mu}(B(x, r))+\delta_{\mu}(B(x, 2 r)) .
\end{aligned}
$$

We claim that

$$
\delta_{\mu}(B(x, r)) \lesssim \min \left(1, \frac{1}{r^{2}}\right) \sin ^{2} \alpha \quad \text { for }(x, r) \notin Z .
$$

In fact, this holds for all $x \in \Gamma$ and $r>0$, but we only need to prove it for $(x, r) \notin Z$. Let us see that the lemma follows from this estimate. We write

$$
\begin{aligned}
\iint_{0}^{\infty} \Delta_{\mu}^{1}(x, r)^{2} \frac{d r}{r} d \mu(x) & \lesssim \iint_{(x, r) \in(\Gamma \times(0, \infty)) \backslash Z} \delta_{\mu}(B(x, r))^{2} \frac{d r}{r} d \mu(x) \\
& \lesssim \sin ^{4} \alpha \iint_{(x, r) \in(\Gamma \times(0, \infty)) \backslash Z} \min \left(1, \frac{1}{r^{4}}\right) \frac{d r}{r} d \mu(x) .
\end{aligned}
$$

So, to prove the lemma it is enough to show that the last integral does not exceed some absolute constant. To this end, denote $\left\{z_{1}\right\}=L_{1} \cap T_{1},\left\{z_{2}\right\}=L_{2} \cap T_{2}$, and $\left\{z_{0}\right\}=T_{1} \cap T_{2}$, and set

$$
B_{1}=\left\{(x, r) \in \Gamma \times(0, \infty): z_{i} \in B(x, 2 r) \text { for some } i=0,1,2 \text { and } T \not \subset B(x, r)\right\}
$$

and

$$
B_{2}=\{(x, r) \in \Gamma \times(0, \infty): x \in T \text { and } T \subset B(x, r)\}
$$


Notice that $\Gamma \times(0, \infty)) \backslash Z=B_{1} \cup B_{2}$ and if $(x, r) \in B_{2}$ then $r>1 / 2$. We then have

$$
\begin{aligned}
\iint_{(x, r) \in(\Gamma \times(0, \infty)) \backslash Z} \min \left(1, \frac{1}{r^{4}}\right) \frac{d r}{r} d \mu(x) \\
=\iint_{B_{1}} \min \left(1, \frac{1}{r^{4}}\right) \frac{d r}{r} d \mu(x)+\iint_{B_{2}} \min \left(1, \frac{1}{r^{4}}\right) \frac{d r}{r} d \mu(x) \\
=\sum_{i=0}^{2} \iint_{\substack{(x, r) \in \Gamma \times(0, \infty) \\
z_{i} \in B(x, 2 r), T \not \subset B(x, r)}} \min \left(1, \frac{1}{r^{4}}\right) \frac{d r}{r} d \mu(x)+\iint_{B_{2}} \min \left(1, \frac{1}{r^{4}}\right) \frac{d r}{r} d \mu(x) .
\end{aligned}
$$

For $0 \leq i \leq 2$ we consider two cases according to whether $r<1 / 2$ or not. We set

$$
\iint_{\substack{(x, r) \in \Gamma \times(0,1 / 2) \\ z_{i} \in B(x, 2 r), T \not \subset B(x, r)}} \min \left(1, \frac{1}{r^{4}}\right) \frac{d r}{r} d \mu(x)=\iint_{\substack{(x, r) \in \Gamma \times(0,1 / 2) \\ z_{i} \in B(x, 2 r), T \not \subset B(x, r)}} \frac{d r}{r} d \mu(x) .
$$

Integrating first with respect to $x$, taking into account that $\left|x-z_{i}\right|<2 r$, the last integral is bounded by

For $r>1 / 2$ we have

$$
c \int_{r \in(0,1 / 2)} r \frac{d r}{r} \approx 1
$$

$$
\iint_{\substack{(x, r) \in \Gamma \times[1 / 2, \infty) \\ z_{i} \in B(x, 2 r), T \not \subset B(x, r)}} \min \left(1, \frac{1}{r^{4}}\right) \frac{d r}{r} d \mu(x)=\iint_{\substack{(x, r) \in \Gamma \times[1 / 2, \infty) \\ z_{i} \in B(x, 2 r), T \not \subset B(x, r)}} \frac{d r}{r^{5}} d \mu(x) .
$$

It is easy to check that for $(x, r)$ in the domain of integration above we have

$$
r-c \leq\left|z_{i}-x\right| \leq r+c
$$

for some absolute constant $c$, which implies that

$$
\mu\left(\left\{x:(x, r) \in \Gamma \times[1, \infty), z_{i} \in B(x, 2 r), T \not \subset B(x, r)\right\}\right) \leq c^{\prime} .
$$

Thus the integral in 4.7 is bounded by

$$
c \int_{r>1 / 2} \frac{d r}{r^{5}} \lesssim 1
$$

Therefore we have shown that

$$
\iint_{B_{1}} \min \left(1, \frac{1}{r^{4}}\right) \frac{d r}{r} d \mu(x) \lesssim 1 .
$$

In the same way, for $r>1 / 2$

$$
\mu\left(\left\{x:(x, r) \in B_{2}\right\}\right) \leq c^{\prime \prime},
$$

hence

$$
\iint_{B_{2}} \min \left(1, \frac{1}{r^{4}}\right) \frac{d r}{r} d \mu(x) \lesssim 1 .
$$

Thus 4.8 and 4.9) imply

$$
\iint_{(x, r) \in(\Gamma \times(0, \infty)) \backslash Z} \min \left(1, \frac{1}{r^{4}}\right) \frac{d r}{r} d \mu(x) \lesssim 1,
$$

as wished. 


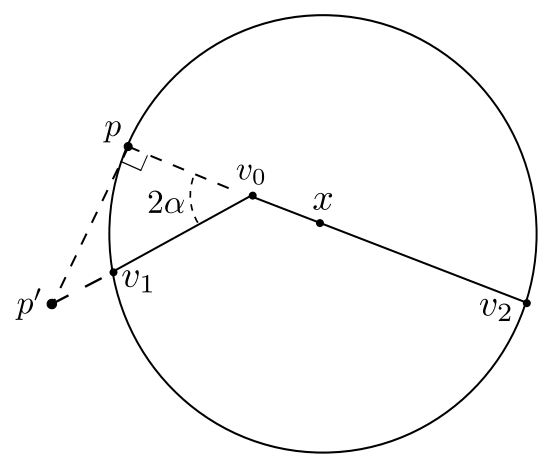

Figure 2. The case $A 1$

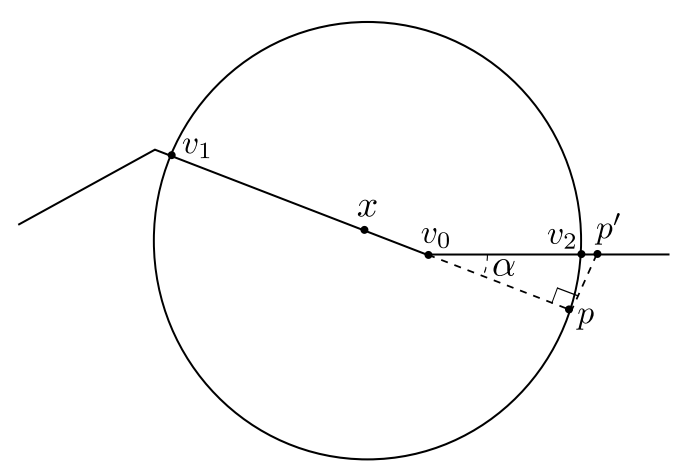

Figure 3 . The case $A 2$

To prove the claim 4.6 we distinguish several cases:

Case A: $(x, r) \notin Z$ and $x \in T$.

Subcase A1: $B(x, 2 r) \cap \Gamma \subset T$.

We note that this subcase is possible only for $r<2$. We write

$$
\begin{aligned}
\delta_{\mu}(B(x, r)) & \leq\left|\frac{\mu(B(x, r))}{r}-2 \cos \alpha\right|+2(1-\cos \alpha) \\
& \approx\left|\frac{\mu(B(x, r))}{r}-2 \cos \alpha\right|+\sin ^{2} \alpha .
\end{aligned}
$$

Since $(x, r) \notin Z$ we have that $B(x, 2 r) \cap T_{i} \neq \varnothing$ for both $i=1,2$. Without loss of generality let $x \in T_{2}$ and set $\left\{v_{0}\right\}=T_{1} \cap T_{2},\left\{v_{i}\right\}=\partial B(x, 2 r) \cap T_{i}$, for $i=1,2$, $\{p\}=\partial B(x, 2 r) \cap L_{v_{0} v_{2}} \backslash\left\{v_{2}\right\}$, where $L_{v_{0} v_{2}}$ denotes the line crossing $v_{0}$ and $v_{2}$. Let also $p^{\prime}$ be the point of intersection of $L_{v_{0} v_{1}}$ and the perpendicular line to $L_{v_{0} v_{2}}$ which passes through $p$. See also Figure 2 .

We have

$$
\begin{aligned}
|\mu(B(x, r))-2 r \cos \alpha| & =\left|\cos \alpha\left(d\left(v_{1}, v_{0}\right)+d\left(v_{0}, v_{2}\right)\right)-\cos \alpha\left(d\left(v_{0}, v_{2}\right)+d\left(v_{0}, p\right)\right)\right| \\
& \left.\approx \mid d\left(v_{1}, v_{0}\right)-d\left(v_{0}, p\right)\right) \mid .
\end{aligned}
$$

Observing that

$$
\begin{aligned}
\left|d\left(v_{1}, v_{0}\right)-d\left(v_{0}, p\right)\right| & \leq d\left(v_{0}, p^{\prime}\right)-d\left(v_{0}, p\right)=d\left(v_{0}, p^{\prime}\right)-d\left(v_{0}, p^{\prime}\right) \cos (2 \alpha) \\
& \approx \sin ^{2}(2 \alpha) d\left(v_{0}, p^{\prime}\right) \lesssim r \sin ^{2} \alpha
\end{aligned}
$$

and recalling 4.10, we deduce that $\delta_{\mu}(B(x, 2 r)) \lesssim \sin ^{2} \alpha$.

Subcase A2: $B(x, 2 r) \cap \Gamma \subset T_{i} \cup L_{i}$ for $i=1$ or 2 .

Without loss of generality we can assume that $i=2$. We consider the following points: $\left\{v_{0}\right\}=L_{2} \cap T_{2},\left\{v_{1}\right\}=\partial B(x, 2 r) \cap T_{2},\left\{v_{2}\right\}=\partial B(x, 2 r) \cap L_{2}$, and $\{p\}=\partial B(x, 2 r) \cap$ $L_{v_{0} v_{1}} \backslash\left\{v_{1}\right\}$. Let also $p^{\prime}$ be the point of intersection of $L_{v_{0} v_{2}}$ and the perpendicular line to $L_{v_{0} v_{1}}$ which passes through $p$. See also Figure 3 . 


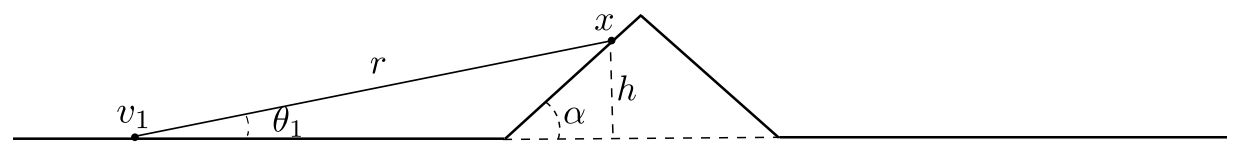

Figure 4. The curve $\Gamma_{\alpha}$.

We have

$$
\begin{aligned}
|\mu(B(x, r))-4 \cos \alpha r| & =\left|\cos \alpha d\left(v_{1}, v_{0}\right)+d\left(v_{0}, v_{2}\right)-\cos \alpha\left(d\left(v_{0}, v_{1}\right)+d\left(v_{0}, p\right)\right)\right| \\
& \leq(1-\cos \alpha) d\left(v_{0}, v_{2}\right)+\cos \alpha\left(d\left(v_{0}, v_{2}\right)-d\left(v_{0}, p\right)\right) \\
& \lesssim r \sin ^{2} \alpha+\cos \alpha\left(d\left(v_{0}, v_{2}\right)-d\left(v_{0}, p\right)\right) .
\end{aligned}
$$

As in the previous subcase,

$$
\begin{aligned}
d\left(v_{0}, v_{2}\right)-d\left(v_{0}, p\right) & \leq d\left(v_{0}, p^{\prime}\right)-d\left(v_{0}, p\right)=d\left(v_{0}, p^{\prime}\right)-d\left(v_{0}, p^{\prime}\right) \cos \alpha \\
& \approx d\left(v_{0}, p^{\prime}\right) \sin ^{2} \alpha \lesssim r \sin ^{2} \alpha,
\end{aligned}
$$

hence we deduce that $\delta_{\mu}(B(x, 2 r)) \lesssim \sin ^{2} \alpha$.

Subcase $A 3: B(x, r) \cap L_{1} \neq \emptyset$ and $B(x, r) \cap L_{2} \neq \emptyset$.

If $r \leq 1$, combining the arguments from the two previous cases, it follows that $\delta_{\mu}(B(x, r)) \lesssim$ $\sin ^{2} \alpha$.

We now consider the case when $r>1$ and without loss of generality we assume that $x=(w, h) \in T_{1}$. Given two lines $L, L^{\prime}$, we denote by $\measuredangle\left(L, L^{\prime}\right)$ the smallest angle between $L$ and $L^{\prime}$. Let $\left\{v_{1}\right\}=\partial B(x, r) \cap L_{1}$ and let $\theta_{1}=\measuredangle\left(L_{x, v_{1}}, L_{1}\right)$. Then it follows easily that

$$
\delta_{\mu}(B(x, r)) \lesssim \sin ^{2} \theta_{1} \approx\left(\frac{h}{r}\right)^{2} \leq \frac{\sin ^{2} \alpha}{r^{2}}
$$

see also Figure 4 .

Case B: $(x, r) \notin Z$ and $x \notin T$.

Let $\left\{v_{1}\right\}=\partial B(x, r) \cap T$, then if $\theta_{1}=\measuredangle\left(L_{x V_{1}}, L_{2}\right)$ we get

$$
\delta_{\mu}(B(x, r))=1-\cos \theta_{1} \approx \sin ^{2} \theta_{1} .
$$

Since $\theta_{1}<\alpha$, we have that $\sin ^{2} \theta_{1}<\sin ^{2} \alpha$. Moreover if $r>1$, as in subcase A3, we get that $\sin ^{2} \theta_{1}<\frac{\sin ^{2} \alpha}{r^{2}}$. Therefore,

$$
\delta_{\mu}(B(x, r)) \lesssim \min \left(1, \frac{1}{r^{2}}\right) \sin ^{2} \alpha .
$$

Thus (4.6) follows and the proof of the lemma is complete.

We wish now to compare the integral $\iint_{0}^{\infty} \Delta_{\mu}^{1}(x, r)^{2} \frac{d r}{r} d \mu(x)$ to the analogous one involving the so called $\beta$-numbers of Peter Jones, which play a key role in the theory of the so called quantitative rectifiability (see [Jo, [DaS1] and [DaS2], for example). Given 
a Radon measure $\mu$ in $\mathbb{R}^{d}$, the Jones' $\beta$-numbers are defined as follows. For $x \in \operatorname{supp}(\mu)$ and $r>0$, set, for $1 \leq p<\infty$,

$$
\beta_{p}^{\mu}(B(x, r))=\inf _{L}\left(\int_{B(x, r)} \frac{\operatorname{dist}(y, L)^{p}}{r^{p+1}} d \mu(y)\right)^{1 / p},
$$

and

$$
\beta_{\infty}^{\mu}(B(x, r))=\inf _{L} \sup _{y \in B(x, r) \cap \operatorname{supp}(\mu)} \frac{\operatorname{dist}(y, L)}{r},
$$

where in both cases the infimum is taken over all lines $L \subset \mathbb{R}^{d}$.

By [Do, Theorem 6] (in the case $1 \leq p<\infty$ ), for the measure $\mu_{\alpha}$ of Proposition 4.1 we have

$$
\iint_{0}^{\infty} \beta_{p}^{\mu_{\alpha}}(B(x, r))^{2} \frac{d r}{r} d \mu_{\alpha}(x) \approx\left\|f^{\prime}\right\|_{2}^{2} \approx \sin ^{2} \alpha
$$

This also holds for $p=\infty$, by [Jo. So together with Proposition 4.1, this yields

$$
\iint_{0}^{\infty} \Delta_{\mu_{\alpha}}^{1}(x, r)^{2} \frac{d r}{r} d \mu_{\alpha}(x) \ll \iint_{0}^{\infty} \beta_{p}^{\mu_{\alpha}}(B(x, r))^{2} \frac{d r}{r} d \mu_{\alpha}(x) \quad \text { as } \alpha \rightarrow 0,
$$

for all $1 \leq p \leq \infty$.

\section{REFERENCES}

[AH] D. R. Adams and L. I. Hedberg, Function spaces and potential theory, Grundlehren Math. Wiss., vol. 314, Springer-Verlag, Berlin 1996.

[CGLT] V. Chousionis, J. Garnett, T. Le and X. Tolsa, Square functions and uniform rectifiability, to appear in Trans. Amer. Math. Soc.

[DaS1] G. David and S. Semmes, Singular Integrals and rectifiable sets in $\mathbb{R}^{n}:$ Au-delà des graphes lipschitziens. Astérisque 193, Société Mathématique de France (1991).

[DaS2] G. David and S. Semmes, Analysis of and on uniformly rectifiable sets, Mathematical Surveys and Monographs, 38. American Mathematical Society, Providence, RI, (1993).

[Do] J.R. Dorronsoro, Mean oscillation and Besov spaces, Canad. Math. Bull., 28 (4) (1985), 474-480.

[ENV] V. Eiderman, F. Nazarov and A. Volberg, Vector-valued Riesz potentials:Cartan-type estimates and related capacities, Proc. Lond. Math. Soc. (3) 101 (2010), no. 3, 727-758.

[EV1] V. Eiderman and A. Volberg, $L^{2}$-norm and estimates from below for Riesz transforms on Cantor sets, Indiana Univ. Math. J., 60 (2011), no. 4, 1077-1112.

[EV2] V. Eiderman and A. Volberg, Non-homogeneous harmonic analysis: 16 years of development, Russ. Math. Surv. (68) 973, (2013).

[JNV] B. Jaye, F. Nazarov and A. Volberg, The fractional Riesz transform and an exponential potential, St. Petersburg Math. J. Vol. 24 (2013), no. 6, 903-938.

[Jo] P.W. Jones, Rectifiable sets and the traveling salesman problem, Invent. Math.102:1, (1990), 1-15.

[M] J.M. Marstrand, The $(\phi, s)$ regular subsets of $n$ space, Trans. Amer. Math. Soc 113 (1964), 369392.

[Ma] P. Mattila, Geometry of sets and measures in Euclidean spaces, Cambridge Stud. Adv. Math. 44, Cambridge Univ. Press, Cambridge, 1995.

[MPV] J. Mateu, L. Prat and J. Verdera, The capacity associated to signed Riesz kernels, and Wolff potentials, J. Reine Angew. Math. 578 (2005), 201-223.

[Na] R. Narasimhan, Introduction to the Theory of Analytic Spaces, Lecture Notes in Mathematics, 25. Springer-Verlag, Berlin, (1966).

[RT] M.C. Reguera and X. Tolsa, Riesz transforms of non-integer homogeneity on uniformly disconnected sets, to appear in Trans. Amer. Math. Soc. 
[T1] X. Tolsa, Principal values for Riesz transforms and rectifiability, J. Funct. Anal., vol. 254(7) 2008, 1811-1863.

[T2] X. Tolsa, Calderon-Zygmund capacities and Wolff potentials on Cantor sets, J. Geom. Anal. 21(1) 2011, 195-223.

[T3] X. Tolsa, Analytic capacity, the Cauchy transform, and non-homogeneous Calderón-Zygmund theory, volume 307 of Progress in Mathematics. Birkhäuser Verlag, Basel, 2014.

[T4] X. Tolsa, Rectifiable measures, square functions involving densities, and the Cauchy transform. Preprint (2014).

[TTo] X. Tolsa and T. Toro, Rectifiability via a square function and Preiss' Theorem, to appear in IMRN.

Vasilis Chousionis. Department of Mathematics, University of Connecticut, Storrs, CT 06269, USA

Department of Mathematics and Statistics, University of Helsinki, P. O. Box 68, Fi-00014, FINLAND

E-mail address: vasileios.chousionis@helsinki.fi

Laura Prat. Departament de Matemàtiques, Universitat Autònoma de Barcelona, CatALONIA

E-mail address: laurapb@mat.uab.cat

Xavier Tolsa. Institució Catalana de Recerca i Estudis Avançats (ICREA) and Departament de Matemàtiques, Universitat Autònoma de Barcelona, Catalonia

E-mail address: xtolsa@mat.uab.cat 